cytes in late prophase I show a premature separation of homologous chromosomes with partial loss of the central element of SC and significantly increased distance between the centromeres. Taken together, our findings indicate that VCL plays an important role in the meiotic progression. We show that the VCL deletion in spermatocytes leads to considerable abnormalities on the level of chromosomal desynapsis which in the end results in the decreased breeding capability of VCL cKO males.

This study is supported by the CSMS scholarship, GACR (16-03403S), the project,BIOCEV“(CZ.1.05/1.1.00/02.0109) and Light Microscopy Core Facility, IMG ASCR.

doi: http://dx.doi.org/10.7124/bc.0009B5

\section{High-resolution study of epigenetic processes: new insights into methylation and demethylation}

\author{
S. Siciliani, I. Masiello, L. Zannino, F. Ba- \\ siricò, C. Casali, E. King, A. Lacavalla, \\ L. Saia, M. Scaltritti, M. Biggiogera \\ Laboratory of Cell Biology and Neurobiology, De- \\ partment of Biology and Biotechnology, University \\ of Pavia, Italy \\ stella.siciliani93@gmail.com
}

Methylation and demethylation are two epigenetic processes of a big relevance for different biological pathways. The two events happen on the carbon in position five of the cytosine belonging to the so called $\mathrm{CpG}$ island. The methylation implies the addition of a methyl group on the cytosine, forming the 5-methylcytosine $(5 \mathrm{mC})$ thanks to enzymes called DNMT (DnaMethyltransferase). After, when required, the methyl group is oxidized or demethylated by a family of enzyme called TET, forming the 5-hydroxymethylcytosine $(5 \mathrm{hmC})$. The role of the $5 \mathrm{mC}$ is generally correlated with gene expression repression, while the $5 \mathrm{hmC}$ function must be clarified. In this context, in order to elucidate the hypothetic role of these markers we decide to investigate at ultrastructural level, by looking at the distribution of two epigenetic modifications putting our attention on different areas of the cell nucleus. Our study where carried out by using transmission electron microscope, light microscope and molecular biology techniques. We observed that in condensed regions of the nucleus the DNA is always highly methylated rather than hydroxymethylated, but in the so called perichromatin region the pattern changes. Indeed, in this region it was possible to notice an abundancy of demethylation underlined both by the presence of the $5 \mathrm{hmC}$ and of the enzymes involved in the processes: TET2. This result could allow to hypothesize a sort of activating role for the oxidized modification respect to its reduced form and underline how the perichromatin region is a dynamic region where DNA status changes.

doi: http://dx.doi.org/10.7124/bc.0009B6

\section{Lamin A and PI(4,5)P2 - a novel complex in the cell nucleus}

Sara Escudeiro-Lopes, Vlada V. Philimonenko, Lenka Jarolimová, and Pavel Hozák

Department of Biology of the Cell Nucleus, Institute of Molecular Genetics ASCR v.v.i. Vídeňská 1083, 142 20, Prague 4, Czech Republic saraescudeiro.l@gmail.com

Lamins are important regulators of nuclear structural integrity as well as nuclear functional processes such as DNA transcription, replication and 
repair, and epigenetic regulation. Mutations in LMNA gene, which encodes for lamin A protein, cause a large variety of human diseases, known as laminopathies, including muscular dystrophies and progeroid syndromes. Phosphatidynositol4,5-bisphosphate (PIP2) is a well described product of the phosphoinositol signalling and it has an important role in splicing and transcription. Here we show that PIP2 is in a complex with lamin A, together with Nuclear myosin 1 (NM1), and lamin A phosphorylation status appears to be implicated in the anchoring of other proteins to the complex, as well as in the movement of lamin A from the nuclear envelope to the nucleoplasm. Thus, we are characterizing this complex using several biochemical and microscopy methods. From our preliminary data we conclude that some Lamin A phosphorylations might be important for PIP2-dependent interactions of lamin A, important for nuclear functions.

We would like to thank to the Grant Agency of the Czech Republic (17-09103S, 16-03346S and 15-08738S) for their financial support to this project. We would also like to thank for the institutional support due to the long-term conceptual development of the scientific organization (RVO: 68378050). The microscopy work was performed at the Microscopy Centre, Institute of Molecular Genetics AS CR, supported by the MEYS CR (LM2015062 Czech Bioimaging).

doi: http://dx.doi.org/10.7124/bc.0009B7

\section{From 3D-Genome Organisation to Cellular and Supra- cellular Morphogenesis}

\section{Klaus Scherrer}

Institut J. Monod, CNRS and Univ. Paris Diderot, klaus.scherrer@ijm.fr
The molecular 3D-model of the "Unified Matrix Hypothesis" (UMH) proposed that the eukaryotic DNA may be organised in a 3D-network and genomic domains, to be transcribed in specific sectors of the nucleus defining "chromosome territories"(1). It generalised by analogy for normal cells the pattern of Ectopic Pairing observed since 1948 in drosophila salivary glands, that links the 4 polytene chromosomes and the nucleolus into a 3D-genomic network. In normal cells where DNA is flexible, an analogous system might operate able to link directly distant sites within and between chromosomes. This putative model at the basis of nuclear architecture and morphogenesis was confirmed by the publication of the $3 \mathrm{D}$ structure of the yeast genome (2) and more recently extended to eukaryotic cells in general (2). The original model of the $\mathrm{UMH}$ also proposed a logical link between cellular and supra-cellular morphogenesis. The 3D-DNA chromatin network organises the nuclear architecture which can be modified back and forth by conversion of hetero- and euchromatin, or by transposition of mobile DNA elements as SINES and LINES or other «jumping genes». In undifferentiated stem cells and de-differentiated cells, having lost their cytoplasm by «shedding», the nucleus occupies most of the cellular volume. In differentiation, it is reduced by retraction of its membrane from the periphery to the center, ending up fully picnotic in terminal differentiation of, e.g. red cells,. In this process, nuclear space becomes cytoplasm leaving behind, possibly, some of its organisation. In the cytoplasm localised synthesis of specific proteins is based on prior 3D-sorting of mRNA; the various types of cytoskeleton are instrumental 